\title{
Significance of vaspin in obstructive sleep apnea-hypopnea syndrome
}

\author{
ZHE PAN, XIANGHUA ZHUANG, XIAOBO LI, SHAOYI HUANG, LIANG ZHANG, \\ FUCHEN LOU, SHIHONG CHEN and YIHONG NI
}

\begin{abstract}
Department of Endocrinology, The Second Hospital of Shandong University, Jinan, Shandong 250033, P.R. China
\end{abstract}
Received October 26, 2014; Accepted November 18, 2015

DOI: $10.3892 /$ etm.2016.2997

\begin{abstract}
Obstructive sleep apnea-hypopnea syndrome (OSAHS) is a commonly-diagnosed chronic sleep disorder. It is considered to be an important independent risk factor in the development of insulin resistance (IR). Patients with OSAHS exhibit a variety of metabolic disorders, including obesity and metabolic syndrome. Visceral adipose tissue-derived serpin (vaspin) is an adipokine that is considered to be a link between obesity and IR. The present study aimed to evaluate the levels of plasma vaspin in patients with OSAHS and examine their potential correlation with sleep characteristics. A total of 20 healthy male subjects and 42 male patients with OSAHS were selected, and patients were divided into mild $(n=22)$ and severe $(n=20)$ OSAHS groups. The 20 patients in the severe OSAHS group received nasal continuous positive airway pressure (nCPAP) treatment for 2 months. Venous blood samples were drawn from all patients in a fasting state prior to and subsequent to nCPAP treatment, which were used to measure the levels of biochemical indicators. The sleep parameters and serologic index changes were compared prior to and following treatment. The values of contractive pressure (SBP), neck circumference (NC), waist circumference (WC), waist-to-hip ratio (WHR), body mass index (BMI) and hip circumference (HC) in the two OSAHS groups were significantly increased compared with those in the control group. In addition, the levels of vaspin in OSAHS patients were markedly increased and vaspin was revealed to be positively associated with fasting blood sugar, fasting insulin, triglycerides, homeostasis model assessment-IR, apnea-hypopnea index (AHI), NC, WC, BMI and WHR $(\mathrm{P}<0.05)$. After 2 months of nCPAP treatment, the SBP and AHI were significantly reduced. In conclusion, vaspin may have an important role in OSAHS patients with
\end{abstract}

Correspondence to: Dr Yihong Ni, Department of Endocrinology, The Second Hospital of Shandong University, 247 Beiyuan Road, Jinan, Shandong 250033, P.R. China

E-mail: nyh6457@163.com

Key words: obstructive sleep apnea-hypopnea syndrome, vaspin, insulin resistance
IR and treatment using nCPAP may improve the condition of OSAHS patients.

\section{Introduction}

Obstructive sleep apnea-hypopnea syndrome (OSAHS) is a common breathing disorder. It is characterized by repetitive collapse of the upper airway during sleep, resulting in impedance to airflow and hypoxia (1). The symptoms of OSAHS include loud snoring, episodes of breathing cessation, gasping and choking sensations that rouse the patient from sleep, nocturia and insomnia (2). Use of nasal continuous positive airway pressure is the primary treatment in adult OSAHS cases (3). The prevalence of OSAHS increases with age and its prevalence in men is significantly higher than that of women of child-bearing age. However, the prevalence in women increases following menopause (4). OSAHS has been reported to be associated with obesity and metabolic syndrome in adults and is an independent risk factor in the development of insulin resistance (IR) (5). Thus far, the underlying mechanisms linking OSAHS and metabolic syndrome remain poorly understood.

Adipokines are a group of biologically active molecules secreted by the white adipose tissue. Adipokines effect a key role in the regulation of glucose and lipid metabolism, as well as in energy homeostasis (6). Visceral adipose tissue-derived serpin (vaspin) is an adipokine that is a member of the serine protease inhibitor family (7). Evidence has demonstrated that vaspin contributes to the development of IR and type 2 diabetes mellitus (8). In the present study, vaspin was hypothesized to be a possible link between metabolic syndrome and OSAHS.

The aim of the present study was to investigate the possible role of vaspin in the pathogenesis of OSAHS. For this purpose, the serum vaspin level in patients with OSAHS was measured prior to and following medical therapy. In addition, the association between serum vaspin and metabolic variables was analyzed.

\section{Materials and methods}

Subjects. The study protocol was approved by the Ethical Committee of the Second Hospital of Shandong University (Jinan, China). All patients provided written informed consent. A total of 42 male patients with OSAHS, aged 34 to 62 years 
old, were enrolled into the study, along with 20 age-matched healthy males that served as the controls. Only male patients were examined in the current study, as gender affects vaspin levels; Körner et al (9) reported that vaspin serum levels were significantly higher in girls than in boys. Furthermore, Li et al (8) revealed that fasting plasma vaspin levels were significantly higher in female compared with male patients in normal glucose tolerance and impaired glucose tolerance subjects. The incidence of OSAHS is higher significantly in men than women, so male patients were selected. These patients were admitted to the Second Hospital of Shandong University between May 2011 and October 2012, with symptoms such as loud snoring, gasping and choking sensations during sleep and insomnia. The diagnosis of OSAHS was established according to the international diagnostic criteria (10). Apnea was defined as episodes of airflow cessation lasting for $>10 \mathrm{sec}$. Hypopnea was defined as $>50 \%$ reduction of airflow below the standard level, in addition to a $4 \%$ decrease in oxyhemoglobin saturation in patients breathing normally during sleep. The sum of time spent in apnea and hypopnea, determined by an overnight sleep study using polysomnography, was divided by the total sleep time in order to determine the apnea-hypopnea index (AHI). Subjects with an AHI of $\geq 5$ were considered to suffer from OSAHS. According to the degree of AHI and the arterial oxygen saturation $\left(\mathrm{SaO}_{2}\right)$, the subjects were divided into two groups: The mild $(n=22)$ and severe $(n=20)$ OSAHS groups. In the mild group, the AHI of patients was between 5 and 20 , and the lowest $\mathrm{SaO}_{2}\left(\mathrm{LSaO}_{2}\right)$ values were between 85 and $89 \%$. In the severe group, the AHI value was $>21$, while the $\mathrm{LSaO}_{2}$ was $<85 \%$. Subjects with diabetes mellitus, cardiovascular and cerebrovascular diseases, chronic renal failure and chronic respiratory diseases were excluded from the study.

Clinical and biochemical measurements. After $12 \mathrm{~h}$ of fasting, the subjects underwent anthropometric evaluation. Their height, weight, neck circumference (NC), waist circumference (WC) and hip circumference (HC) were measured by a trained nurse. Body mass index (BMI) was calculated as the body weight in kilograms divided by the square of the height in metres [BMI = weight $(\mathrm{kg}) /$ height $\left.^{2}(\mathrm{~m})\right]$. The waist-to-hip ratio (WHR) was defined as the WC divided by the HC. Systolic blood pressure (SBP) and diastolic blood pressure (DBP) were also measured.

After $12 \mathrm{~h}$ of fasting, 5-6 $\mathrm{ml}$ venous blood was collected from each of the patients, prior and subsequent to treatment. The samples were centrifuged at 1,000-1,200 $\mathrm{x} g$ and kept at $-80^{\circ} \mathrm{C}$ until required for subsequent assays. The level of fasting plasma glucose (FPG) was measured by the glucose-oxidase method (11). In addition, fasting insulin (FINS) was assessed using a human insulin-specific radioimmunoassay kit (Linco Research, Inc., St. Louis, MO, USA). Triglyceride (TG) levels, total cholesterol (TC), high-density lipoprotein (HDL) cholesterol and low-density lipoprotein cholesterol were measured using the UniCel DxC 800 analyzer (Beckman Coulter, Inc., Brea, CA, USA). Furthermore, the vaspin and adiponectin (APN) levels were measured using human Serpin A12/Vaspin quantikine and human total adiponectin/Acrp30 quantikine ELISA kits (R\&D Laboratories, Inc., Antrim, UK), respectively, according to the manufacturer's instructions. The intra-assay (describing the variation of results within the data set) and interassay coefficients of variation (describing the variation of results from repeated experiments) for vaspin and adiponectin were $9 \%$ and $11 \%$, respectively. The IR index was then calculated by homeostasis model assessment (HOMA-IR) based on the FPG and insulin concentrations using the equation: HOMA-IR = FPG $(\mathrm{mmol} / \mathrm{l}) \times$ FINS $(\mathrm{mIU} / \mathrm{l}) / 22.5$

Treatment of severe OSAHS group. The 20 patients in the severe OSAHS group received nasal continuous positive airway pressure (nCPAP) treatment for 2 months, but the mild OSAHS group received no treatment. nCPAP treatment was selected as the most effective and least invasive treatment method. Prior to the treatment, the patients were informed regarding the details and necessity of the treatment. Subsequently, the patients were treated using an automatic voltage regulator ventilator (ResMed S8 AutoSet II; ResMed, San Diego, CA, USA) at the hospital for 1 night. The patients were treated with continuous ventilation using a nasal mask, which ensured that the patient's respiratory tract remained unobstructed at night in order to correct the apnea and reduce the hypoxic effects. Patients were taught to use the equipment by a trained specialist nurse. Patients then underwent a night of nCPAP treatment, during which a number of parameters were determined with polysomnography. The patients almost all required long-term treatment at home for at least $4 \mathrm{~h}$ per night, and these were followed-up for 2 months. An E-series polysomnographer (Compumedics Ltd., Abbotsford, Australia) was used to measure and record the overnight polysomnographic parameters, including an electroencephalogram (in order to distinguish the sleep stage), electrocardiogram (in order to record changes in the heart rate), oronasal airflow (in order to analyze the presence of apnea), chest and abdominal movements by strain gauges, and pulse oxygen saturation. After 2 months of treatment and follow-up, the 20 patients with severe OSAHS were subjected to the same clinical and biochemical measurements as prior to treatment.

Statistical analysis. All data are presented as the mean \pm standard deviation, and were analyzed using SPSS 18.0 software (SPSS, Inc., Chicago, IL, USA). Prior to analysis, the data were assessed for normality of distribution using the Shapiro-Wilk test. The data were analyzed using one-way analysis of variance, Pearson's correlation and stepwise multiple regression analysis. A value of $\mathrm{P}<0.05$ was considered to indicate a statistically significant difference.

\section{Results}

Baseline characteristics of the patients. The baseline characteristics of the controls and OSAHS patients are listed in Table I. Within the total of 62 male patients included in the present study, 42 of these suffered from OSAHS. The mild group included 22 cases, the severe group included 20 cases, and 20 males were enrolled in the control group. No statistically significant differences were observed in age between the three groups $(\mathrm{P}>0.05)$. Compared with the control group, the values of SBP, DBP, NC, WC, WHR, BMI and $\mathrm{HC}$ in the two OSAHS groups were significantly increased $(\mathrm{P}<0.05)$. Compared with the mild OSAHS group, the WC, BMI and $\mathrm{HC}$ in the severe OSAHS group were considerably increased, 
Table I. Baseline characteristics of controls and patients with OSAHS (mean \pm standard deviation).

\begin{tabular}{|c|c|c|c|}
\hline Characteristics & Control group $(n=20)$ & $\begin{array}{l}\text { Mild OSAHS } \\
\text { group }(n=22)\end{array}$ & $\begin{array}{c}\text { Severe OSAHS } \\
\text { group }(n=20)\end{array}$ \\
\hline Mean age (years) & $46.75 \pm 11.19$ & $47.90 \pm 12.23$ & $49.25 \pm 11.42$ \\
\hline $\mathrm{SBP}(\mathrm{mmHg})$ & $130.25 \pm 8.92$ & $133.58 \pm 11.35^{\mathrm{a}}$ & $134.96 \pm 12.45^{\mathrm{a}}$ \\
\hline DBP (mmHg) & $72.28 \pm 6.78$ & $78.61 \pm 10.50^{\mathrm{a}}$ & $80.31 \pm 8.15^{\mathrm{a}}$ \\
\hline $\mathrm{NC}(\mathrm{cm})$ & $35.73 \pm 1.37$ & $37.70 \pm 2.31^{\mathrm{a}}$ & $38.72 \pm 3.62^{\mathrm{a}}$ \\
\hline $\mathrm{WC}(\mathrm{cm})$ & $82.31 \pm 6.85$ & $94.19 \pm 7.64^{\mathrm{a}}$ & $98.23 \pm 8.49^{\mathrm{a}, \mathrm{b}}$ \\
\hline WHR (cm) & $0.84 \pm 0.08$ & $0.93 \pm 0.06^{\mathrm{a}}$ & $0.95 \pm 0.08^{\mathrm{a}}$ \\
\hline BMI & $24.28 \pm 1.52$ & $25.91 \pm 2.43^{\mathrm{a}}$ & $27.11 \pm 1.87^{\mathrm{a}, \mathrm{b}}$ \\
\hline $\mathrm{HC}(\mathrm{cm})$ & $97.06 \pm 9.35$ & $101.33 \pm 8.83^{\mathrm{a}}$ & $103.12 \pm 9.62^{\mathrm{a}, \mathrm{b}}$ \\
\hline AHI (times/h) & $3.30 \pm 1.03$ & $11.00 \pm 2.61^{\mathrm{a}}$ & $29.95 \pm 8.15^{\mathrm{a}, \mathrm{b}}$ \\
\hline Average $\mathrm{SaO}_{2}(\%)$ & $97.10 \pm 1.55$ & $87.27 \pm 1.42^{\mathrm{a}}$ & $78.30 \pm 5.01^{\mathrm{a}, \mathrm{b}}$ \\
\hline FPG $(\mathrm{mmol} / \mathrm{l})$ & $4.88 \pm 0.28$ & $5.00 \pm 0.46$ & $5.24 \pm 0.32^{\mathrm{a}, \mathrm{b}}$ \\
\hline FINS (mU/l) & $13.12 \pm 1.71$ & $15.99 \pm 1.31^{\mathrm{a}}$ & $17.91 \pm 2.63^{\mathrm{a}, \mathrm{b}}$ \\
\hline TG (mmol/l) & $1.19 \pm 0.27$ & $1.66 \pm 0.29^{a}$ & $1.90 \pm 0.27^{\mathrm{a}, \mathrm{b}}$ \\
\hline $\mathrm{TC}(\mathrm{mmol} / \mathrm{l})$ & $4.57 \pm 0.47$ & $4.70 \pm 0.50$ & $5.09 \pm 0.67^{\mathrm{a}, \mathrm{b}}$ \\
\hline $\mathrm{HDL}(\mathrm{mmol} / \mathrm{l})$ & $1.57 \pm 0.22$ & $1.52 \pm 0.26$ & $1.35 \pm 0.39^{\mathrm{a}, \mathrm{b}}$ \\
\hline LDL (mmol/l) & $2.46 \pm 0.51$ & $2.42 \pm 0.43$ & $2.87 \pm 0.62^{\mathrm{a}, \mathrm{b}}$ \\
\hline HOMA-IR & $2.84 \pm 0.26$ & $3.56 \pm 0.46^{\mathrm{a}}$ & $4.17 \pm 0.48^{\mathrm{a}, \mathrm{b}}$ \\
\hline Vaspin (ng/ml) & $1.48 \pm 0.25$ & $1.78 \pm 0.45^{\mathrm{a}}$ & $1.85 \pm 0.32^{\mathrm{a}}$ \\
\hline $\mathrm{APN}(\mathrm{ng} / \mathrm{ml})$ & $10.53 \pm 2.36$ & $8.63 \pm 1.43^{\mathrm{a}}$ & $8.77 \pm 1.34^{\mathrm{a}}$ \\
\hline
\end{tabular}

${ }^{\mathrm{a}} \mathrm{P}<0.05$ vs. control group; ${ }^{\mathrm{b}} \mathrm{P}<0.05$ vs. mild group. OSAHS, obstructive sleep apnea-hypopnea syndrome; SBP, systolic blood pressure; DBP, diastolic blood pressure; NC, neck circumference; WHR, waist-to-hip ratio; BMI, body mass index; AHI, apnea-hypopnea index; $\mathrm{SaO}_{2}$, arterial oxygen saturation; FPG, fasting plasma glucose; FINS, fasting insulin; TG, triglycerides; TC, total cholesterol; HDL, high-density lipoprotein cholesterol; LDL, low-density lipoprotein cholesterol; HOMA-IR, homeostasis model assessment-insulin resistance; vaspin, visceral adipose tissue-derived serpin; APN, adiponectin; WC, waist circumference; HC, hip circumference.

while no significant difference in SBP, DBP, NC or WHR was observed between the two OSAHS groups. The AHI was significantly increased in the mild and severe groups compared with the control group, and between these OSAHS groups $(\mathrm{P}<0.05)$. The average $\mathrm{SaO}_{2}$ was significantly decreased in the severe group compared with the control and mild groups, and between the control and mild groups $(\mathrm{P}<0.05)$. In addition, the FPG level in the severe OSAHS group was significantly higher compared with that of the mild OSAHS and control groups $(\mathrm{P}<0.05)$, while the FPG level in the mild OSAHS group was not significantly increased compared with that in the control group $(\mathrm{P}>0.05)$. Serum FINS, TG levels and HOMA-IR index were significantly increased compared with the control group in the mild and severe groups, and in the severe group compared with the mild group. TC and LDL levels were increased and HDL levels decreased in the severe OSAHS group compared with the mild and the control groups $(\mathrm{P}<0.05)$, but no significant difference was observed in levels of these between the control and mild groups ( $>0.05)$. The level of vaspin in the mild and severe OSAHS groups $(1.78 \pm 0.45$ vs. $1.85 \pm 0.32 \mathrm{ng} / \mathrm{ml}$, respectively) was significantly higher compared with that in the control group $(1.48 \pm 0.25 \mathrm{ng} / \mathrm{ml} ; \mathrm{P}<0.01)$; however, no significant difference was observed between the mild and severe groups $(\mathrm{P}>0.05)$. APN levels were significantly higher in the control group compared with the two OSAHS groups $(\mathrm{P}<0.05)$.
Study of serum vaspin levels. Based on the results of Pearson's correlation analysis, the level of vaspin was positively associated with WC, FPG, FINS, HOMA-IR, TG, NC, BMI, WHR, AHI and $\mathrm{NC}(\mathrm{P}<0.05)$, and negatively associated with $\mathrm{SaO}_{2}$ and $\mathrm{APN}$ level (Table II). Therefore, vaspin was defined as the dependent variable, and WC, WHR, BMI, FPG, FINS, HOMA-IR, TG, AIH, APN and $\mathrm{SaO}_{2}$ were defined as the independent variables in order to perform multiple linear regression analysis. Multiple stepwise regression analysis showed that HOMA-IR and $\mathrm{SaO}_{2}$ were independent risk factors that influenced the vaspin levels $\left(\mathrm{Y}=2.875-0.021 \mathrm{SaO}_{2}+0.588 \times\right.$ HOMA-IR; Table III).

Comparison of values in severe group prior to and following 2 months of nCPAP treatment. After 2 months of nCPAP treatment, the SBP, AHI and vaspin were significantly reduced and $\mathrm{SaO}_{2}$ and APN were increased compared with the values prior to treatment $(\mathrm{P}<0.05)$. Other indices were not significantly altered (Table IV).

\section{Discussion}

Sleep apnea syndrome accounts for $70 \%$ of sleep disorder cases, with OSAHS being the most common type of this syndrome (12). OSAHS can occur at any age and gender, but is most prevalent in males with an age of $>40$ years. In addition, obesity is an important factor causing upper airway 
Table II. Pearson's correlation analysis between vaspin and other variables.

\begin{tabular}{|c|c|c|}
\hline Characteristics & $\mathrm{r}$ & P-value \\
\hline WC & 0.286 & 0.048 \\
\hline FPG (mmol/l) & 0.291 & 0.022 \\
\hline FINS (mU/l) & 0.640 & $<0.001$ \\
\hline HOMA-IR & 0.627 & $<0.001$ \\
\hline TG (mmol/l) & 0.604 & $<0.001$ \\
\hline BMI & 0.462 & $<0.001$ \\
\hline WHR & 0.465 & $<0.001$ \\
\hline AHI (times/h) & 0.595 & $<0.001$ \\
\hline $\mathrm{NC}(\mathrm{cm})$ & 0.520 & 0.001 \\
\hline Average $\mathrm{SaO}_{2}(\%)$ & -0.667 & $<0.001$ \\
\hline $\mathrm{APN}(\mathrm{ng} / \mathrm{ml})$ & -0.398 & 0.001 \\
\hline
\end{tabular}

Vaspin, visceral adipose tissue-derived serpin; $r$, correlation coefficient; WC, waist circumference; FPG, fasting plasma glucose; FINS, fasting insulin; HOMA-IR, homeostasis model assessment-insulin resistance; TG, triglycerides; NC, neck circumference; BMI, body mass index; WHR, waist-to-hip ratio; AHI, apnea-hypopnea index; $\mathrm{SaO}_{2}$, arterial oxygen saturation; APN, adiponectin.

Table III. Impact factors of vaspin through multiple stepwise regression analysis.

\begin{tabular}{lrccc}
\hline Model & B-value & SE & t-test & P-value \\
\hline Constant & 2.875 & 0.876 & 3.283 & 0.002 \\
$\mathrm{SaO}_{2}$ & -0.021 & 0.007 & -3.198 & 0.002 \\
HOMA-IR & 0.588 & 0.284 & 2.068 & 0.043 \\
\hline
\end{tabular}

Vaspin, visceral adipose tissue-derived serpin; SE, standard error; $\mathrm{SaO}_{2}$, arterial oxygen saturation; HOMA-IR, homeostasis model assessment-insulin resistance.

blockage (13). In the present study, subjects were enrolled into three groups, including the mild OSAHS, severe OSAHS and control groups. The mean age of the three groups was not significantly different $(\mathrm{P}>0.05)$. The BMI, NC, WC and WHR in the OSAHS groups were markedly higher compared with the values in the control group. Therefore, OSAHS appears to be closely associated with obesity-related indicators. However, obese patients do not necessarily suffer from OSAHS, while certain patients with OSAHS have a normal BMI (14). In the current study, OSAHS patients were found to have an imbalance in body fat distribution, since the $\mathrm{NC}, \mathrm{WC}$ and WHR in the OSAHS groups were higher compared with those in control subjects with the same BMI. Increased NC indicated that excess fat was deposited around the upper airway, and increased WHR indicated that superfluous fat was deposited in the viscus. Thus, weight reduction may be useful to relieve and treat OSAHS.

OSAHS has been shown to be an independent risk factor of abnormal glucose and lipid metabolism (15). Meslier et al (16) showed that the incidence of type 2 diabetes mellitus and
Table IV. Comparison of severe group prior to and following nCPAP treatment.

\begin{tabular}{lcc}
\hline Index & $\begin{array}{c}\text { Prior to } \\
\text { treatment }\end{array}$ & $\begin{array}{c}\text { Following } \\
\text { treatment }\end{array}$ \\
\hline $\mathrm{SBP}(\mathrm{mmHg})$ & $134.96 \pm 12.45$ & $129.27 \pm 10.18^{\mathrm{a}}$ \\
$\mathrm{DBP}(\mathrm{mmHg})$ & $80.31 \pm 8.15$ & $80.25 \pm 6.20$ \\
$\mathrm{AHI}$ & $29.95 \pm 8.15$ & $18.20 \pm 6.45^{\mathrm{a}}$ \\
Average $\mathrm{SaO}{ }_{2}$ & $78.30 \pm 5.01$ & $85.68 \pm 6.02^{\mathrm{a}}$ \\
FPG $(\mathrm{mmol} / \mathrm{l})$ & $5.24 \pm 0.32$ & $5.20 \pm 0.39$ \\
$\mathrm{FINS}(\mathrm{mU} / \mathrm{l})$ & $17.91 \pm 2.63$ & $16.52 \pm 2.07$ \\
TG $(\mathrm{mmol} / \mathrm{l})$ & $1.90 \pm 0.27$ & $1.81 \pm 0.36$ \\
TC $(\mathrm{mmol} / \mathrm{l})$ & $5.09 \pm 0.67$ & $5.10 \pm 0.82$ \\
$\mathrm{HDL}(\mathrm{mmol} / \mathrm{l})$ & $1.35 \pm 0.39$ & $1.36 \pm 0.53$ \\
$\mathrm{LDL}(\mathrm{mmol} / \mathrm{l})$ & $2.87 \pm 0.62$ & $2.67 \pm 0.49$ \\
$\mathrm{HOMA}-\mathrm{IR}$ & $4.17 \pm 0.48$ & $4.09 \pm 0.57$ \\
Vaspin $(\mathrm{ng} / \mathrm{ml})$ & $1.85 \pm 0.32$ & $1.61 \pm 0.27^{\mathrm{a}}$ \\
APN $(\mathrm{ng} / \mathrm{ml})$ & $8.77 \pm 1.34$ & $10.15 \pm 1.8^{\mathrm{a}}$ \\
\hline
\end{tabular}

${ }^{\mathrm{a}} \mathrm{P}<0.05$ vs. value prior to the nCPAP treatment. nCPAP, nasal continuous positive airway pressure; SBP, systolic blood pressure; DBP, diastolic blood pressure; AHI, apnea-hypopnea index; $\mathrm{SaO}_{2}$, arterial oxygen saturation; FPG, fasting plasma glucose; FINS, fasting insulin; TG, triglycerides; TC, total cholesterol; HDL, high-density lipoprotein cholesterol; LDL, low-density lipoprotein cholesterol; HOMA-IR, homeostasis model assessment-insulin resistance; vaspin, visceral adipose tissue-derived serpin; APN, adiponectin.

impaired glucose tolerance in OSAHS males was 30.1 and $20.0 \%$, respectively. In the present study, all subjects had a normal FPG level (3.9-6.0 mmol/l). However, the FPG level in the severe OSAHS group was higher compared with that of the control group $(\mathrm{P}<0.05)$ and non-significantly higher than that in the mild group $(P>0.05)$. This might be due to the small sample size used in the present study. The levels of FPG, FINS, HOMA-IR, TG and TC in the OSAHS groups were higher than those in the control group. The HOMA-IR index in the OSAHS groups was positively correlated with BMI and AHI, and negatively correlated with $\mathrm{SaO}_{2}$. Therefore, the OSAHS treatment selected should be able to regulate glucose metabolism and prevent the development of diabetes.

As mentioned earlier, OSAHS was shown to be closely associated with obesity-related indicators. Adipose tissue function may play an important role in the pathogenesis of OSAHS. In addition to neutral fat storage, energy storage, energy supply and temperature regulation, adipose tissue is also an endocrine organ with the function of endocrine, autocrine and paracrine signaling (17). Adipose tissue is able to secrete $>100$ types of adipokines, including leptin, APN, tumor necrosis factor- $\alpha$, interleukin- 6 and vaspin. Adipose tissue also plays an important role in IR and body metabolism (18). Hida et al (15) originally identified the new adipokine, vaspin, in visceral adipose tissue of Otsuka Long-Evans Tokushima Fatty rats, and vaspin was found to be part of the serpin superfamily (19). In the present study, the level of serum vaspin was measured and a significant difference was identified between the control and OSAHS groups. The level of vaspin was also found to be 
associated with BMI, WL, WHR, TG, HOMA-IR and FINS. Therefore, we speculate that vaspin may be associated with the glucose metabolism, IR and obesity indices. In addition, the level of serum vaspin was positively associated with AHI $(\mathrm{P}<0.05)$ and negatively associated with $\mathrm{SaO}_{2}(\mathrm{P}<0.05)$; thus, we hypothesize that vaspin is associated with the severity of OSAHS and degree of nocturnal hypoxia. Vaspin was then defined as a dependent variable and multiple linear regression analyses were performed. As shown in the results, $\mathrm{SaO}_{2}$ and HOMA-IR were independent of vaspin. Therefore, vaspin is considered to be positively correlated with IR and OSAHS.

A principal and potential damage of OSAHS is the disturbance of the normal sleep structure (20). Microarousal is a neurological event, during which sleep fragmentation is activated, including the autonomic nervous system and multiple neural networks (21). Intermittent hypoxia and sleep fragmentation can activate the sympathetic-adrenal axis (22). In the short-term, this activation can inhibit insulin excretion and stimulate hepatic glucose output. Furthermore, insulin sensitivity in peripheral tissues may be reduced, resulting in IR (23). If left untreated, OSAHS may lead to cardiovascular and cerebrovascular diseases, as well as a variety of complications (22).

nCPAP is an efficient strategy and the main method used to treat OSAHS, with efficiency rates that can reach 70-80\%. As shown in numerous studies, subsequent to nCPAP, the time of apnea was reduced, hypoxia was corrected, IR was improved and metabolic disorders were recovered $(24,25)$. Furthermore, the treatment can improve the blood metabolism, coronary atherosclerotic heart disease and high blood pressure.

In conclusion, in the present study, the levels of blood pressure, TGs and FINS were decreased following nCPAP treatment in the severe OSAHS group, although the changes were not statistically significant. The non-significant result may be due to the short time of nCPAP treatment. Nevertheless, a beneficial effect of nCPAP treatment in OSAHS patients was observed.

\section{Acknowledgements}

This study was funded by the Second Hospital of Shandong University (grant no. Y2013010017).

\section{References}

1. Sunitha $\mathrm{C}$ and Aravindkumar S: Obstructive sleep apnea: Clinical and diagnostic features. Indian J Dent Res 20: 487-491, 2009.

2. Banno K and Kryger MH: Sleep apnea: Clinical investigations in humans. Sleep Med 8: 400-426, 2007.

3. Chen X, Niu X, Xiao Y, Dong J, Lu M and Kong W: Effect of continuous positive airway pressure on leptin levels in patients with obstructive sleep apnea: A meta-analysis. Otolaryngol Head Neck Surg 152: 610-618, 2015.

4. Riha RL, Gislasson T and Diefenbach K: The phenotype and genotype of adult obstructive sleep apnoea/hypopnoea syndrome. Eur Respir J 33: 646-655, 2009.

5. Tamada D, Otsuki M, Kashine S, Hirata A, Onodera T, Kitamura T and Shimomura I: Obstructive sleep apnea syndrome causes a pseudo-Cushing's state in Japanese obese patients with type 2 diabetes mellitus. Endocr J 60: 1289-1294, 2013.
6. Trujillo ME and Scherer PE: Adipose tissue-derived factors: Impact on health and disease. Endocr Rev 27: 762-778, 2006.

7. Hida K, Wada J, Eguchi J, Zhang H, Baba M, Seida A, Hashimoto I, Okada T, Yasuhara A, Nakatsuka A, et al: Visceral adipose tissue-derived serine protease inhibitor: A unique insulin-sensitizing adipocytokine in obesity. Proc Natl Acad Sci USA 102: 10610-10615, 2005.

8. Li K, Li L, Yang M, Liu H, Liu D, Yang H, Boden G and Yang G: Short-term continuous subcutaneous insulin infusion decreases the plasma vaspin levels in patients with type 2 diabetes mellitus concomitant with improvement in insulin sensitivity. Eur J Endocrinol 164: 905-910, 2011

9. Körner A, Neef M, Friebe D, Erbs S, Kratzsch J, Dittrich K, Blüher S, Kapellen TM, Kovacs P, Stumvoll M, et al: Vaspin is related to gender, puberty and deteriorating insulin sensitivity in children. Int J Obes 35: 578-86, 2011.

10. Dacal Quintas R, Tumbeiro Novoa M, Alves Pérez MT, Santalla Martínez ML, Acuña Fernández A and Marcos Velázquez P: Obstructive sleep apnea in normal weight patients: Characteristics and comparison with overweight and obese patients. Arch Bronconeumol 49: 513-517, 2013.

11. Kingsley GR and Getchell G: Direct ultramicro glucose oxidase method for determination of glucose in biologic fluids. Clin Chem 6: 466-475, 1960

12. Chokroverty S: Overview of sleep \& sleep disorders. Indian J Med Res 131: 126-140, 2010.

13. Iguchi A, Yamakage H, Tochiya M, Muranaka K, Sasaki Y, Kono S, Shimatsu A and Satoh-Asahara N: Effects of weight reduction therapy on obstructive sleep apnea syndrome and arterial stiffness in patients with obesity and metabolic syndrome. J Atheroscler Thromb 20: 807-820, 2013.

14. Kelishadi R, Nilforoushan N, Okhovat A, Amra B, Poursafa P and Rogha M: Effects of adenoidectomy on markers of endothelial function and inflammation in ormal-weight and overweight prepubescent children with sleep apnea. J Res Med Sci 16 (Suppl 1): S387-S394, 2011.

15. Hida K, Wada J, Zhang H, Hiragushi K, Tsuchiyama Y, Shikata K and Makino $\mathrm{H}$ : Identification of genes specifically expressed in the accumulated visceral adipose tissue of OLETF rats. J Lipid Res 41: 1615-1622, 2000.

16. Meslier N, Gagnadoux F, Giraud P, Person C, Ouksel H, Urban T and Racineux JL: Impaired glucose-insulin metabolism in males with obstructive sleep apnoea syndrome. Eur Respir J 22: 156-160, 2003.

17. Harwood HJ Jr: The adipocyte as an endocrine organ in the regulation of metabolic homeostasis. Neuropharmacology 63: 57-75, 2012

18. Pérez PM, Moore-Carrasco R, González DR, Fuentes EQ and Palomo IG: Gene expression of adipose tissue, endothelial cells and platelets in subjects with metabolic syndrome (Review). Mol Med Rep 5: 1135-1140, 2012.

19. Wada J: Vaspin: A novel serpin with insulin-sensitizing effects. Expert Opin Investig Drugs 17: 327-333, 2008.

20. Pedrosa RP, Krieger EM, Lorenzi-Filho G and Drager LF: Recent advances of the impact of obstructive sleep apnea on systemic hypertension. Arq Bras Cardiol 97: e40-e47, 2011 (In English, Portuguese, Spanish).

21. Douglas NJ and Martin SE: Arousals and the sleep apnea/hypopnea syndrome. Sleep 19: S196-S197, 1996.

22. Zamarrón C, Valdés Cuadrado L and Alvarez-Sala R: Pathophysiologic mechanisms of cardiovascular disease in obstructive sleep apnea syndrome. Pulm Med: 521087, 2013.

23. Lurie A: Metabolic disorders associated with obstructive sleep apnea in adults. Adv Cardiol 46: 67-138, 2011.

24. Ruhle KH, Domanski U and Nilius G: Obstructive pressure peak: A new method for differentiation of obstructive and central apneas under auto-CPAP therapy. Sleep Breath 17: 111-115, 2013.

25. Oyama J, Yamamoto H, Maeda T, Ito A, Node K and Makino N: Continuous positive airway pressure therapy improves vascular dysfunction and decreases oxidative stress in patients with the metabolic syndrome and obstructive sleep apnea syndrome. Clin Cardiol 35: 231-236, 2012. 